\title{
Archives
}

\section{Sortir de la Grande Noirceur grâce aux documents d'archives}

\section{Jacques Rouillard}

Volume 48, numéro 1, 2019

$46^{\mathrm{e}}$ Congrès de l'Association des archivistes du Québec

URI : https://id.erudit.org/iderudit/1060817ar

DOI : https://doi.org/10.7202/1060817ar

Aller au sommaire du numéro

Éditeur(s)

Association des archivistes du Québec (AAQ)

ISSN

0044-9423 (imprimé)

2369-9256 (numérique)

Découvrir la revue

\section{Citer cet article}

Rouillard, J. (2019). Sortir de la Grande Noirceur grâce aux documents d'archives. Archives, 48(1), 107-143. https://doi.org/10.7202/1060817ar

\section{Résumé de l'article}

L'objectif de ce texte est de montrer l'importance de l'acquisition, de la conservation et du classement des documents d'archives pour cerner notre histoire et éviter que le récit historique devienne une construction mythique sans fondement véritable. Les bibliothécaires et les archivistes sont en quelque sorte les gardiens de la mémoire d'une société. Il appartient aux historiens de consulter ces documents pour comprendre et interpréter le passé.

Malheureusement, ce n'est pas ce cheminement qui s'est imposé dans la représentation de l'histoire du Québec contemporain qui domine encore notre mémoire collective, selon laquelle le Québec francophone d'avant 1960 est une société monolithique et ultraconservatrice, qui serait entrée de plain-pied dans la modernité à l'occasion de la Révolution tranquille. Cette vision du passé, portée par des intellectuels impatients d'en finir avec le régime de Maurice Duplessis et influencée par une lecture de l'histoire du Québec mise de l'avant par plusieurs sociologues, repose sur une recherche historique très sommaire. Elle est remise en question à partir des années 1970 par des historiens intéressés à l'histoire du Québec récent, dont la formation et la méthodologie les portent à accorder une importance primordiale à la consultation de fonds d'archives. Leurs travaux apportent un nouvel éclairage sur l'histoire du Québec depuis la Confédération. Loin d'être une société figée où règne l'unanimité sociale, il apparaît que le pouvoir clérical est contrebalancé par d'autres forces venant des milieux politiques et syndicaux. Il en ressort l'image d'une société, sans doute distincte, mais aussi diversifiée. C'est ce que l'auteur montre à partir de travaux qu'il a réalisés depuis plus de quarante ans portant sur divers aspects de l'histoire du Québec. La représentation misérabiliste du passé sous le signe de la Grande Noirceur et de l'impuissance est un mythe qui affecte la psychologie collective des Québécois et Québécoises, les porte à rejeter une large portion de leur passé et contribue à ternir leur sens d'identité.
Ce document est protégé par la loi sur le droit d'auteur. L'utilisation des services d’Érudit (y compris la reproduction) est assujettie à sa politique d'utilisation que vous pouvez consulter en ligne.

https://apropos.erudit.org/fr/usagers/politique-dutilisation/ 


\section{EONGRÈS DE L'AAQ}

\section{Sortir de la Grande Noirceur grâce aux documents d'archives}

\section{JACQUES ROUILLARD, PROFESSEUR ÉMÉRITE}

Département d'histoire, Université de Montréal

Lors de la réception du prix Jacques-Grimard au congrès 2017 de l'Association des archivistes du Québec, nous avons remercié l'auditoire en évoquant que nous avions une dette de reconnaissance envers les archivistes: "Sans votre travail, je n'aurais pu remettre en question la vision de I'histoire du Québec contemporain issue des artisans de la Révolution tranquille qui présente le Québec comme une société ultraconservatrice, monolithique, tournée sur elle-même. » C'est ce cheminement à travers divers fonds d'archives que nous voulons évoquer dans ce texte en montrant comment la consultation de documents écrits et de journaux d'époque m'a amené à réviser ma perception de l'histoire du Québec contemporain. Nous ne sommes pas les seuls dans cette barque: plusieurs jeunes historiens dans les années 1970 en arrivent à des conclusions similaires. Nous nous appliquons d'abord à montrer comment s'est imposée dans les années 1950 et 1960 une interprétation qui domine encore notre mémoire collective à partir d'une connaissance sommaire de notre histoire. 


\section{LE QUÉBEC À L'HEURE DE LA GRANDE NOIRCEUR}

Nous avons lu récemment un volume intitulé Le cœur des Québécois. L'évolution du Québec de 1976 à aujourd'hui où les auteurs tracent « un nouveau portrait du Québec contemporain » en « déboulonnant certains mythes» (Grégoire, Montigny et Rivest, 2016, Page de couverture). Il est destiné à faire connaître la société québécoise depuis 1976. Les auteurs consacrent quelques pages à un "petit retour en arrière » où les Québécois, écrivent-ils, vivaient jusqu'au milieu du XXe siècle dans une période de survivance où les nationalistes développaient une société traditionnelle conservatrice. Les élites politiques et religieuses prêchaient l'obéissance aux institutions et visaient l'accroissement du nombre par la natalité. Elles prônaient l'appartenance à l'ethnie, I'homogénéité et aux valeurs comme la piété et l'humilité. Leur diffusion plaçait davantage la société québécoise «en position de repli qu'en phase d'émancipation » (Grégoire, Montigny et Rivest, 2016, p. 45). Évidemment, le dépli survient avec la Révolution tranquille où «l'État se modernise et remplace l'Église en tant qu'institution centrale de la société » (Grégoire, Montigny, Rivest, 2016, p. 45-46).

Nous faisons référence à ce volume, instructif à plusieurs autres égards, pour décrire la représentation qui est largement dominante de notre passé au sein de la population. Nous pourrions citer de nombreux autres ouvrages, articles de revue et de journaux qui véhiculent fréquemment la même interprétation de l'histoire du Québec. Notre mémoire collective a complètement intériorisé l'idée que nos ancêtres ont fait fausse route et qu'ils ont vécu dans une «Grande Noirceur» jusqu'à la Révolution tranquille. Avec comme conséquence le rejet d'une large portion de notre histoire, un peu plus d'un siècle (1850-1960), perçue comme une période « honteuse » de notre histoire. II va sans dire que cette construction colore aussi grandement l'opinion que les Canadiens anglais se font de l'histoire du Québec, pour qui la province a été une «priest ridden province» responsable de son passé malheureux.

Cette représentation n'est pas sans conséquence. La mémoire de son passé fait partie des fondements de l'identité tout autant pour un individu que pour une collectivité. Que cette expérience ait été positive ou négative, elle affecte de manière consciente ou inconsciente l'opinion de soi et joue un rôle dans les choix à poser pour l'avenir. D'autre part, I'identité collective se définit pour beaucoup en comparaison et en 
opposition avec les traits, passés et présents, des autres sociétés qui I'entourent. Une perception négative de son expérience par rapport à celle des autres sociétés entame la fierté de ses membres et contribue à dévaloriser l'appartenance à cette collectivité, notamment pour ceux et celles qui ont le choix de la joindre ou non.

L'historien Jocelyn Létourneau a mené récemment une enquête scientifique auprès de jeunes Québécois sur leur conscience de leur passé. II s'en dégage que la majorité des jeunes francophones détient une vision malheureuse de son passé qui est fait de «rendez-vous ratés avec I'histoire». Ces échecs seraient, selon lui, « un des socles sur lequel s'élève l'identitaire franco-québécois» (Létourneau, 2014, p. 174). Cette sombre image de leur histoire ne s'enracine pas uniquement dans la lutte pour la survie du Québec comme collectivité; l'épisode raté de son histoire jusqu'à la Révolution tranquille contribue à une image négative que les Québécois et Québécoises se renvoient d'eux-mêmes. Elle concourt à la dévaluation de leur société avec pour conséquence un manque de fierté et d'assurance collectives. C'est désolant, et ce l'est encore davantage, car cette représentation de notre passé est inexacte quand on base la recherche historique sur la consultation de documents qui portent sur l'ensemble des forces qui ont modelé la construction de la société québécoise.

La tendance naturelle des gens est de projeter leur analyse de la société dans laquelle ils vivent en imaginant son passé à l'aune du présent et en faisant abstraction du contexte changeant dans lequel elle évolue. C'est ce qui est arrivé au Québec dans les années 1950 et 1960 alors que, dans les milieux intellectuels, on se désole du long règne de I'Union nationale (1939-1960) et que I'on devient impatient de moderniser le Québec selon le modèle des autres sociétés industrialisées. Se greffe une vision de l'ensemble de l'histoire du Québec qui dépeint son évolution sous le signe d'un nationalisme réactionnaire.

\section{L'INTERPRÉTATION SOCIOLOGIQUE}

Cette construction de I'histoire du Québec doit beaucoup à des sociologues qui se sont penchés sur la société québécoise et qui ont proposé une lecture de son histoire dès les années 1950. À l'époque, les rares historiens professionnels dans les universités québécoises se 
désintéressent de l'histoire du Québec contemporain, parce qu'ils croient manquer du recul nécessaire pour effectuer une recherche valable sur les dernières décennies. Ils laissent ce champ aux spécialistes des sciences sociales, dont les sociologues qui vont effectivement présenter leur version de son histoire.

De par leur formation, ces derniers cherchent à comprendre les problèmes sociaux au moment de leur déroulement et ils s'efforcent d'insérer leurs observations dans un cadre théorique basé sur de vastes explications des événements. Ils ont tendance à vouloir appliquer un modèle d'interprétation développé par un illustre représentant de leur discipline et à vérifier s'il s'applique au sujet de leur recherche. Leurs travaux sur le Québec après la Deuxième Guerre mondiale consistent d'abord en des enquêtes sur le terrain, surtout sur les communautés rurales, mais aussi sur le monde urbain qu'on présente comme bouleversé par le développement industriel.

Pour assoir leur compréhension du Québec, ils y greffent une vision de son histoire qui repose sur une très mince couche documentaire. Leur formation ne les porte pas à mener une recherche érudite en archives ou à dépouiller, par exemple, des journaux d'époque. Ils présentent donc l'histoire de la société franco-québécoise en transposant sur son passé la lecture critique qu'ils font de la société qui les entoure. Depuis, à tout le moins, l'échec des rébellions de 1837 et 1838, ils la dépeignent comme une société organique, dominée par le clergé catholique et dotée de valeurs traditionnelles en décalage avec les forces issues de I'industrialisation. On retrouve parmi eux des sociologues des universités Laval et de Montréal comme Maurice Tremblay, Jean-Charles Falardeau, Fernand Dumont, Marcel Rioux, Hubert Guindon, etc. ${ }^{1}$. Les élites se méfieraient de l'industrialisation et développeraient une profonde rancœur envers I'urbanisation (Dumont, 1965, p. 13).

Leur vision est fortement influencée par le modèle imaginé par des sociologues de l'École de Chicago voulant que le passage de la folk society à la urban society entraîne une modernisation des valeurs à mesure qu'une société s'urbanise et s'industrialise. Deux sociologues attachés à cette école, Horace Miner et Everett Hughes (il enseigne à I'Université McGill de 1927 à 1938), ont fait enquête au Québec durant l'entredeux-guerres pour étudier la transition ruralité/urbanité à Saint-Denis et à Drummondville. Leur maître à penser, Robert Redfield, écrit même 
que la société des paysans canadiens-français, à cause de son isolement, « ressemble aux peuples primitifs» et se compare "d'un certain point de vue », «à celles des Indiens d'Amérique ou des indigènes africains » (Rioux et Martin, 1971, p. 69-70). Ils développent l'idée que l'Église catholique, qui domine la structure sociale du Québec, bloque sa transition vers la modernité liée à l'industrialisation.

Nos sociologues québécois reprennent ce modèle expliquant que le cléricalisme et le duplessisme illustrent effectivement le retard du Québec à se moderniser comme l'ont réalisé les autres sociétés nord-américaines. Les transformations de la Révolution tranquille marqueraient finalement la fin de la Grande Noirceur, la sortie d'une mentalité rétrograde et l'entrée des francophones de plain-pied dans la modernité. Elle comblerait ainsi son retard avec les autres sociétés nord-américaines.

Dans les années 1950, d'autres milieux adoptent la même lecture de I'histoire du Québec, en même temps qu'ils critiquent le conservatisme de leur société. En milieu intellectuel, on retrouve des revues comme Cité libre, L'Action nationale et le journal Le Devoir après qu'un nouveau directeur, Gérard Filion, lui fasse prendre une nouvelle orientation en 1947. La critique rejoint le milieu artistique avec le manifeste Refus global, rédigé en 1948 par Paul-Émile Borduas et ses compagnons, qui dénoncent l'obscurantisme de leur société. En littérature est célébrée la fin du roman du terroir remplacé par le réalisme social du milieu urbain de romans comme Bonheur d'occasion de Gabrielle Roy en 1945 ou Les Plouffe de Roger Lemelin en 1948, adapté pour la télévision et qui connaît un immense succès dans les années 1950 .

Le monde du travail n'est pas en reste avec le collectif dirigé par Pierre Elliott Trudeau sur la grève de l'amiante de 1949 où il trace un réquisitoire percutant contre la pensée sociale des Canadiens français, qui serait imprégnée «de méfiance de l'industrialisation, de repliement sur soi et de nostalgie terrienne » (Trudeau, 1956, p. 88). Reprenant une interprétation qui prend forme dans des articles du Devoir et de Cité libre au début des années 1950, il considère la grève comme « un épisode-clé d'émancipation sociale» (Trudeau, 1956, p. 401) qui marquerait, dans la foulée de la poussée industrielle de l'après-guerre, l'irruption de la classe ouvrière dans I'histoire du Québec. À son point de vue, c'est une force nouvelle qui s'affirme, porteuse de changement social profond qui ébranle l'emprise des élites traditionnelles. Le volume est à la source 
de l'interprétation voulant que cette grève a eu pour effet d'extirper le Canada français d'un profond conservatisme et de le propulser dans le monde moderne.

Dès les années 1950, deux explications relevant de I'histoire prévalent pour expliquer ce retard des Franco-Québécois. L'une, articulée notamment par Pierre Elliot Trudeau, veut que les Canadiens français n'aient jamais cru à la démocratie, qu'ils n'aient pas vraiment joué le jeu du fédéralisme canadien et qu'ils soient eux-mêmes responsables de leur infériorité économique, politique et culturelle. L'autre en fait porter le blâme sur la Conquête de 1760 et la condition de nation colonisée qui a suivi sa dépendance. II ne serait alors que normal que les Québécois se libèrent de la tutelle du Canada anglais en accédant à I'indépendance politique.

\section{L'INTERPRÉTATION HISTORIQUE}

Cette vision qui présente la Révolution tranquille comme une rupture dans la trame historique du Québec a été mise en cause par plusieurs historiens, dont nous sommes, à partir des années 1970. Au milieu des années 1960, il y a moins de cinq professeurs spécialisés en histoire canadienne dans les départements d'histoire des universités de Montréal et de Québec. Certains font porter leur enseignement et leur recherche sur I'histoire de la Nouvelle-France, d'autres sur les années du régime britannique et quelques-uns s'attardent aux décennies qui suivent la Confédération, étudiées dans le cadre de l'ensemble du Canada avec une attention spéciale pour le Canada français.

La composition des cours offerts va changer dans les années 1970 avec l'embauche d'un plus grand nombre de professeurs d'histoire aux universités Laval et de Montréal, ainsi que la création de l'Université du Québec et de ses constituantes. C'est la conséquence de la réforme du système d'éducation et la formation des cégeps qui permettent un accès facilité aux études universitaires. Ces années coïncident également avec la montée du nationalisme avec pour conséquence l'apparition de séminaires sur l'histoire du Québec plutôt que du Canada et l'arrivée de professeurs qui veulent faire porter leur recherche sur I'histoire du Québec contemporain. C'est le cas, par exemple, des professeurs Jean Hamelin et Claude Galarneau au département d'histoire de I'Université 
Laval et de René Durocher au département d'histoire de l'Université de Montréal. De jeunes historiens embauchés dans le réseau de I'Université du Québec suivront cette avenue.

Leurs travaux, qui reposent sur une recherche historique beaucoup plus fouillée, montrent que le Québec s'industrialise et s'urbanise depuis le début du $X X^{e}$ siècle à un rythme similaire à celui de la province voisine. Ce n'est pas qu'ils soient plus perspicaces que les sociologues ou autres intellectuels de sciences sociales, mais la méthodologie de leur discipline met davantage l'accent sur une bonne érudition avant l'établissement d'une interprétation plus générale.

À partir du début des années 1980, ils peuvent aussi tirer profit d'un meilleur accès aux documents avec les nouveaux services offerts par les Archives nationales du Québec (ANQ) qui sont centralisés au pavillon Casault sur le campus de I'Université Laval. L'obtention de microfilms touchant l'état civil, les actes notariés, les recensements, le registre de la population et les index du palais de justice facilite la consultation et fait exploser la fréquentation au pavillon Casault à partir de 1982 (Lessard, 2017). S'ajoute la Loi sur les archives de 1983 (RLRQ, chapitre A-21.1) qui oblige le dépôt des documents conservés par les ministères et l'ouverture de centres des Archives nationales du Québec (ANQ) dans neuf régions du Québec en 1981 (Héon, 1995, p. 10-12). Pour faciliter le travail de recherche des historiens, de nombreux ouvrages de référence sont mis à leur disposition dans les années 1970 (répertoires, journaux sur microfilms, index, bibliographies, etc.). Personnellement, nous avons beaucoup tiré profit des périodiques québécois microfilmés de la Société canadienne du microfilm qui deviennent graduellement disponibles dans les bibliothèques universitaires.

Toujours est-il que les travaux de plusieurs historiens à partir des années 1970 apportent un nouvel éclairage à l'histoire du Québec contemporain. La Révolution tranquille apparaît moins comme une rupture pour la société francophone que comme un moment d'accélération de son évolution. Le processus d'industrialisation et son corollaire, l'urbanisation, ont touché de façon significative la population francophone depuis le milieu du XIX ${ }^{e}$ siècle. Loin d'être une société figée où règne l'unanimité sociale, il leur apparaît que le clergé catholique n'occupe pas une place aussi prépondérante dans le champ social et que son pouvoir est contrebalancé par d'autres forces 
sociales venant des milieux politiques et syndicaux qui sont porteurs de valeurs différentes.

Nous n'allons pas développer plus avant les conclusions de ces travaux, ce que nous avons fait ailleurs dans un article publié en 1998 (Rouillard, 1998, p. 23-51) $)^{2}$. Nous en retenons uniquement que la société francophone est traversée par un vigoureux courant libéral qui anime les gens d'affaires et le monde politique. Leur idéologie en est venue à occuper un espace assez vaste pour faire contrepoids au cléricoconservatisme. Leur libéralisme s'affirme surtout sous une forme modérée à travers les deux grandes formations politiques au Québec jusqu'à l'élection de I'Union nationale en 1936, les partis libéral et conservateur. Au début du XX $X^{e}$ siècle, leur vision est défendue par les grands quotidiens francophones proches du Parti libéral (La Presse, La Patrie, Le Soleil) qui détiennent des tirages considérables, loin devant les quotidiens cléricoconservateurs. Pendant la Deuxième Guerre mondiale, I'administration libérale d'Adélard Godbout (1939-1944) revoit, comme nous le verrons, l'orientation du parti afin de le situer dans le courant social-démocrate.

Cette vision de l'histoire du Québec se retrouve dans la première solide synthèse sur I'histoire du Québec contemporain, celle de René Durocher, Paul-André Linteau, Jean-Claude Robert et François Ricard, publiée en deux volumes en 1979 et $1986^{3}$. Une autre synthèse plus récente, celle de Peter Gossage et J. I. Little, parue d'abord en anglais en 2012, partage la même interprétation; les auteurs sont soucieux de mettre en relief la diversité idéologique et politique du Québec contemporain (Gossage et Little, 2015, p. 159-297).

Mais voilà que, dans les années 1990, des critiques s'élèvent reprochant à ces historiens de présenter le Québec d'avant la Révolution tranquille comme une société "normale » à l'image des autres sociétés nordaméricaines en minimisant l'influence des idées et de la religion (Rudin, 1992 , p. 30-61; 1995, p. 9-42; Kelly, 2003, p. 1-51, 73-106). II y a parmi eux des partisans de la thèse du monolithisme idéologique qui reviennent à l'image de la folk society qui prévalait chez les sociologues des années 1950 et 1960. C'est le cas d'historiens chevronnés tels Gérard Bouchard pour qui l'Église catholique domine sans partage la pensée des élites francophone pendant cent ans, de l'échec des rébellions à 1940. Comme il ignore complètement la pensée issue du monde économique, politique et de larges pans de la société, le Canada français 
lui apparaît avoir sombré dans «l'impuissance collective», fermé dans un conservatisme réactionnaire, réfractaire à l'industrialisation alors que les autres collectivités du Nouveau Monde manifesteraient une vision conquérante animée de force et de liberté (Bouchard, 2000, p. 156, $403 ; 2004$, p. 248) ${ }^{4}$.

Les deux derniers volumes de synthèse d'Yvan Lamonde sur I'histoire des idées depuis 1929 ne tiennent plus compte du libéralisme modéré $d^{\prime}$ inspiration britannique qui anime les intellectuels et la classe politique et qu'il met en évidence dans ses ouvrages antérieurs (Lamonde, 2011, 2016). Ce courant disparaît du paysage pour expliquer l'avènement de la modernité au Québec qui est à l'origine de la Révolution tranquille. Sous I'influence du philosophe français Jacques Maritain, le virage serait attribuable au courant de pensée personnaliste qui anime la revue Cité libre, l'École sociologique de Laval, le journal Le Devoir, les leaders des mouvements d'action catholique et du syndicalisme catholique, les signataires du Refus global, etc ${ }^{5}$.

Dans son dernier essai récent traçant un bilan de ses années de recherche, Lamonde révèle la "fine fleur» de ses réflexions présentant plus de cent ans de notre histoire sous le signe du nationalisme culturel de conservation qui unit le pouvoir politique et religieux. Le Québec francophone est défini comme une société bloquée, divisée, impuissante, inachevée, toujours fatiguée à cause finalement du «trauma» du colonialisme britannique qui «a réussi à empêcher la souveraineté du soi et du nous » (Lamonde, 2017, p. 104). Comme société à l'expérience ratée, on ne saurait trouver meilleur exemple.

\section{CHEMINEMENT PERSONNEL AU CONTACT DES ARCHIVES}

Nous nous permettons maintenant d'être critique envers cette vision de l'histoire du Québec issue des années 1950 qui ne concorde pas du tout avec les résultats de nos recherches depuis les années 1970 en fréquentant divers centres d'archives. C'est à ce cheminement auquel nous nous attardons maintenant.

En 1968, un jeune professeur du département d'histoire de l'Université Laval, Jean Hamelin, décide de consacrer son séminaire de maîtrise à I'histoire des travailleurs et travailleuses au Québec. C'était la première 
fois qu'un historien, professeur d'université, s'intéressait à ce sujet de recherche. Influencé par l'École historique française des Annales, il ambitionne avec I'historien Fernand Ouellet de rédiger une histoire globale du Québec en privilégiant les dimensions économique et sociale. Ouellet, qui s'est réservé la période 1760-1850, en fait le sujet de sa fameuse thèse de doctorat publiée en 1966 sous le titre: Histoire économique et sociale du Québec, 1760-1850 (Ouellet, 1966).

Jean Hamelin s'engage à assumer la même approche pour la période 1850-1896 pour laquelle il associe son collègue Yves Roby (Hamelin et Roby, 1971). À la fin des années 1960, il embauche donc des étudiants pour dépouiller les principaux journaux de la fin du XIX ${ }^{\mathrm{e}}$ siècle, ce qui ne peut se faire à l'époque qu'à la bibliothèque du Parlement de Québec. Les informations colligées lui font réaliser que le Québec s'industrialise pendant cette période et que la société québécoise se transforme beaucoup plus profondément qu'il ne le croyait. Bien sûr, qui dit démarrage industriel, dit aussi travailleurs salariés et naissance d'une classe ouvrière qu'on disait absente de la société québécoise avant la poussée industrielle de la Deuxième Guerre mondiale. Les journaux font voir que des grèves surviennent un peu partout au Québec et que les travailleurs se regroupent en syndicats. Hamelin décide alors d'organiser en 1968 un séminaire de maîtrise sur l'histoire des travailleurs et travailleuses de la période qu'il étudie, en remettant aux étudiants les fiches accumulées par ses assistants l'été précédent pour que chacun d'entre eux présente un travail d'une trentaine de pages.

Nous sommes du nombre et, en collaboration avec une étudiante, nous avons rédigé un travail sur le mouvement ouvrier de 1851 à 1896 qui nous a fait réaliser l'importance de deux organisations syndicales venues des États-Unis: les assemblées de Chevaliers du travail et les syndicats rattachés aux unions internationales. Hamelin réunit les travaux des étudiants dans deux publications: Répertoire des grèves dans la province de Québec au XIX siècle et Les travailleurs québécois, 1851-1896 (Hamelin, Larocque et Rouillard, 1970; Noël, 1973). Nous lui demandons de diriger notre mémoire de maîtrise qu'il nous propose de faire porter sur les grèves importantes survenues dans les filatures de coton au Québec au début du XXe siècle. Notre travail de recherche va nous familiariser avec les nombreuses publications du ministère fédéral du Travail depuis 
1900 et les archives que le ministère a versées à Bibliothèque et Archives Canada (BAC) à Ottawa (Rouillard, 1974).

Pendant I'été 1969, Hamelin nous embauche pour recueillir des informations sur le mouvement ouvrier à partir de journaux de 1896 à 1914, période sur laquelle il compte faire travailler les étudiants inscrits à son séminaire de maîtrise. En dépouillant le quotidien La Presse d'une facture tout à fait nouvelle et que l'éditeur destine aux lecteurs du milieu ouvrier, nous découvrons l'existence d'une chronique ouvrière journalière qui rend compte des informations concernant principalement les nombreux syndicats affiliés aux unions internationales à Montréal.

Nous préparons des fiches dont plusieurs portent sur la montée d'un parti ouvrier animé par les leaders des syndicats internationaux. Réalisant que le sujet est tout à fait nouveau, Hamelin nous demande de rédiger un texte à partir de ces fiches. Nous acceptons et traçons un historique du Parti ouvrier de sa fondation de 1899 à 1915 qui présente une dizaine de candidats aux élections fédérales et provinciales à Montréal dont un est élu en 1906, Alphonse Verville. Le programme du Parti ouvrier, qui est inspiré de celui du Parti travailliste de Grande-Bretagne (Labour Party), propose le suffrage universel, l'éducation gratuite et obligatoire, la nationalisation ou la municipalisation des entreprises de services publics (téléphone, électricité, tramways, chemins de fer), I'assurance maladie, l'assurance vieillesse, l'impôt progressif sur le revenu, etc. Ses adversaires, hommes politiques et membres du clergé, l'accusent pour cette raison d'être un parti socialiste.

Hamelin trouve le texte suffisamment intéressant pour l'inclure dans le recueil portant sur les idéologies au Canada français de 1900 à 1929 qu'il édite avec Fernand Dumont, Fernand Harvey et Jean-Paul Montminy (Rouillard, 1974, p. 267-312). Notre historique tranche avec les autres textes d'étudiants qui portent plutôt sur l'analyse de périodiques se situant dans le courant idéologique clérico-conservateur. Présentant un survol de la période en début de volume, Dumont cite des extraits de la lettre pastorale du cardinal Bégin du diocèse de Québec en 1923 pour conclure qu'en ce début d'industrialisation, le Canada français montre "les attitudes d'une population étrangère à son devenir », manifestant trois dominantes: "repli sur soi-même, repli sur la terre, repli sur le passé » (Dumont, Hamelin, Harvey et Montminy, 1974, p. 8). Quant à notre texte, il prend soin de noter en bas de page qu'il s'agit « d'une très 
belle étude », mais que ce mouvement, qui est porteur d'un programme «venant d'ailleurs», «n'a guère eu d'impact sur l'espace idéologique québécois» (Dumont, Hamelin, Harvey, Montminy, 1974, p. 7). Pour les raisons évoquées plus tôt, Dumont ne peut imaginer le Canada français autrement que sous le signe du conservatisme clérical.

Désireux de poursuivre nos études, nous nous inscrivons aux études doctorales au département d'histoire à l'Université d'Ottawa en 1971 sous la direction de Joseph Levitt qui s'est spécialisé sur le nationalisme d'Henri Bourassa. Nous lui proposons de faire porter le sujet de notre thèse sur les relations entre syndicalisme et nationalisme en traitant de la naissance et du développement des syndicats catholiques au Québec de 1900 à 1930. Ce sujet nous permettait de mettre à profit nos connaissances sur les syndicats internationaux dont l'expansion provoque la formation de syndicats catholiques à partir de 1912. Au cours de nos recherches, nous tirons profit notamment de documents manuscrits conservés à $B A C$, à Bibliothèque et Archives nationales du Québec (BAnQ), au Service d'archives de l'Université Laval, etc. Notre thèse, qui a été publiée, révèle encore une fois I'ampleur du syndicalisme international au Québec et s'attarde à la naissance de syndicats canadiens non confessionnels, puis confessionnels, pour lui faire échec (Rouillard, 1979).

Embauché au département d'histoire de l'Université York de Toronto en 1975, puis à celui de I'Université de Montréal en 1978, nous avons poursuivi nos recherches et publications sur le syndicalisme québécois et sur d'autres sujets, car la consultation de documents et de journaux nous révélait une tout autre vision du Québec que celle articulée par les sociologues des années 1950 et 1960. Pour la faire ressortir, nous avons débordé de l'histoire syndicale pour traiter de sujets que nous examinons plus bas, soit le sens à donner à la Révolution tranquille, la pensée libérale des politiciens, la signification de la devise du Québec et les motifs qui ont présidé à la construction du pont de Québec.

\section{L'APPORT DU SYNDICALISME}

Dans un article publié en 1983, nous avons montré à partir de données statistiques recueillies annuellement par le ministère fédéral du Travail que les taux de syndicalisation au Québec depuis 1901 à 1970 sont comparables à ceux de la province d'Ontario et des États-Unis quand on 
tient compte des populations respectives de salariés (Rouillard, 1983, p. 201-225). De même, l'activité de grève ne se distingue guère de celle de la province voisine, lorsque mesurée en termes de jours de travail perdus à cause de conflits de travail. Le militantisme ouvrier au Québec est particulièrement intense de 1917 à 1922. Pour la seule année 1919, il y a une centaine de conflits de travail qui représentent 750000 jours ouvrables perdus. Cette année record ne sera dépassée que beaucoup plus tard, en 1966, à l'époque des grèves des secteurs public et parapublic et à un moment aussi où le nombre de salariés est beaucoup plus imposant. Parmi ces arrêts de travail, il y en a plusieurs qui sont beaucoup plus significatifs et fructueux pour les travailleurs que la grève de l'amiante de 1949. Cette dernière est malheureusement une grève perdue selon les dires mêmes du principal acteur du conflit, Rodolphe Hamel, qui dresse un bilan de la grève en 1974 (Rouillard, 1999, p. 33-43; 2000, p. 307-342; Hamel, 1974). II devient alors bien difficile de soutenir la thèse que les travailleurs québécois seraient des modèles de docilité avant la Deuxième Guerre mondiale ou qu'ils accuseraient un «retard » à s'éveiller à l'action collective.

Plusieurs de nos travaux mettent notamment en relief l'importance beaucoup plus importante des unions internationales (c'est le terme largement utilisé à l'époque) parmi les syndiqués québécois que les syndicats catholiques (Rouillard, 1989; 2004). En 1911, on compte déjà 20000 membres de syndicats internationaux au Québec, 55000 en 1931, aux trois quarts francophones. Ils devancent sensiblement le nombre de syndiqués catholiques, dont les effectifs fluctuent entre le quart et le tiers des syndiqués (Rouillard, 2001, p. 13-14). Chaque premier lundi de septembre, ils organisent à Montréal, de 1886 à 1953, un défilé de la Fête du travail qui réunit des milliers de travailleurs syndiqués avec pour objectif de manifester symboliquement la fierté de leur travail, la force du syndicalisme et leur identité comme classe sociale (Rouillard, 2010, p. 33-73). Un de leurs leaders, Gustave Francq, fonde un hebdomadaire, Le Monde ouvrier, qui devient le porte-parole des syndicats internationaux et qui est encore publié de nos jours en tant qu'organe de la Fédération des travailleurs et travailleuses du Québec (FTQ) (Rouillard, 2011, p. 19-26).

Les syndicats internationaux ne limitent pas leur action à la négociation collective. Dès la fin du XIX siècle, ils se dotent d'une structure $d^{\prime}$ intervention auprès des trois niveaux de gouvernement. À partir de 
1890, une délégation soumet chaque année au gouvernement du Québec un cahier de revendications tirées de résolutions adoptées démocratiquement. Leurs réclamations de 1900 à 1930 portent sur un large éventail de sujets qui vont bien au-delà des relations du travail: l'éducation gratuite et obligatoire, l'âge minimal de travail à 16 ans, la nationalisation des entreprises de services publics (chemins de fer, tramways, électricité, etc.) et la mise en place d'un filet de sécurité sociale (pensions de vieillesse, assurance chômage, assurance maladie, etc.). Elles concernent aussi l'élargissement des droits démocratiques comme l'abolition du Sénat et du Conseil législatif, le droit de vote pour les femmes et l'élimination du cens d'éligibilité pour les candidats à l'échevinage et à la mairie de Montréal. Ils sont porteurs d'un projet social-démocrate de société qui repose sur un renforcement des valeurs démocratiques et une intervention accrue de l'État en économie et dans le domaine de la sécurité sociale.

Signe de l'importance accordée aux revendications syndicales, la rencontre annuelle des représentants de syndicats internationaux se déroule en présence du premier ministre, de plusieurs ministres et députés. Dans les années 1920, c'est souvent le Cabinet des ministres au complet qui accueille la délégation. Le gouvernement du Québec, administré par le Parti libéral de 1897 à 1936 et de 1939 à 1944, se montre à l'écoute des revendications et adopte plusieurs de leurs réclamations (Rouillard, 2009, p. 111-135, 233-264). En revanche, celui de Duplessis, qui dirige la province de 1936 à 1939 et de 1944 à 1960, situe son action dans une tout autre optique. C'est d'ailleurs pour le combattre que les syndicats internationaux fondent, en 1937, la Fédération provinciale du travail du Québec, ancêtre de l'actuelle FTQ. Elle a pour principale mission de faire échec aux lois antisyndicales du régime Duplessis et de les défendre des accusations de sympathie communiste (Rouillard, 1990, p. 346-374).

\section{LES ARCHIVES SYNDICALES}

Pour que l'apport du syndicalisme en général en vue d'améliorer le sort économique et social des travailleurs et travailleuses soit reconnu dans notre histoire, nous avons participé en 1972 à la fondation du Regroupement des chercheurs en histoire des travailleurs et travailleuses 
du Québec (RCHTQ). Le groupe a pour objectif de favoriser l'enseignement et la recherche en histoire des travailleurs québécois et de promouvoir la diffusion de travaux sur ce sujet. Nous nous sommes rendu compte aussi rapidement qu'il fallait y joindre la conservation des documents syndicaux, car l'histoire des travailleurs ne peut s'écrire si on ne se soucie pas de récupérer, d'inventorier et de rendre disponibles les documents qui les concernent (Rouillard, 1974, p. 32-38; 1974, p. 67-69; 1978, p. $125-130 ; 1986$, p. 3).

Des membres du RCHTQ entreprennent alors d'établir des inventaires de documents conservés par des organisations syndicales et participent à la classification de documents pour en assurer leur conservation. C'est le cas surtout de la documentation d'organismes syndicaux affiliés à la Confédération des syndicats nationaux (CSN) à Trois-Rivières, Rimouski, Québec, Chicoutimi et dans le Nord-Ouest québécois, projet financé souvent avec des fonds provenant de BAnQ. D'autres projets de conservation aboutissent à des inventaires. En 1981, sous la direction de professeurs de l'Université du Québec à Montréal, cinq étudiants de cette institution dressent un inventaire numérique du fonds d'archives conservé au Centre de documentation de la CSN à Montréal (5 000 dossiers).

Devenu professeur au département d'histoire de l'Université de Montréal, nous obtenons en 1985 les fonds nécessaires pour inventorier les documents conservés par les syndicats internationaux à Montréal, sensibiliser la direction de ces syndicats à l'importance de conserver leurs archives et les inciter à faire le dépôt de leurs documents dans des centres d'archives. Ce projet, pour lequel nous avons obtenu une subvention du Conseil de recherche en sciences humaines du Canada, aboutit en 1987 à la publication du Guide des archives des syndicats internationaux qui recense les documents conservés par 46 syndicats internationaux à Montréal (Cardin et Rouillard, 1987). Toujours est-il que l'intérêt pour plusieurs chercheurs envers I'histoire syndicale s'est traduit par l'acquisition de fonds de syndicats par les centres d'archives de plusieurs universités et que bon nombre d'étudiants ont complété leur mémoire de maîtrise ou thèse de doctorat sur ce sujet.

Cette attention des chercheurs s'est atténuée depuis les années 1990, de sorte que le souci de récupération et de conservation des archives syndicales s'est affaibli. II faut ajouter aussi que les services d'archives des 
universités limitent maintenant leur fonction à recueillir leurs documents administratifs et que BAnQ acquiert parfois des fonds syndicaux, mais sans pouvoir, à court terme, les classer et les rendre disponibles aux chercheurs. Mais voilà qu'est formé, en 2013, le Centre d'histoire et d'archives du travail (CHAT), qui s'est donné comme mission de préserver la mémoire des syndicats québécois et d'organismes en lien avec le monde du travail. Réunissant au départ des retraités de syndicats affiliés à la CSN et à la FTQ et des professeurs d'université, il s'efforce par la suite de recruter des membres individuels et institutionnels. Au début 2013, le Centre a emménagé dans un local fourni à Montréal par les Métallurgistes unis d'Amérique et il a embauché temporairement un archiviste et une historienne stagiaire. La même année, le Bureau de la FTQ a invité ses syndicats affiliés à contribuer financièrement au démarrage du CHAT et le congrès de la FTQ a approuvé une résolution en sa faveur afin d'assurer la transmission de I'histoire du mouvement.

En 2014, le Centre a adopté une politique d'acquisition des archives et a commencé à recevoir et à inventorier des fonds de syndicats classés par un archiviste. Plus d'une vingtaine de fonds sont maintenant accessibles aux chercheurs. La liste de ces fonds et leur inventaire se trouvent sur le site Web du Centre qui offre aussi les services d'un archiviste aux syndicats désireux de conserver et de rendre accessibles leurs documents dans leurs bureaux ${ }^{6}$. Le CHAT s'est doté également d'un centre de documentation sur le monde du travail et publie un Bulletin d'information trimestriel. C'est un heureux développement pour enrichir la mémoire du syndicalisme et montrer qu'il a contribué non seulement à améliorer le sort des salariés, mais qu'il a façonné depuis longtemps l'évolution du Québec bien avant la Deuxième Guerre mondiale.

\section{LA RÉVOLUTION TRANQUILLE: RUPTURE OU TOURNANT?}

Notre consultation de fonds d'archives, notamment des ministères du Travail à BAnQ et à BAC, nous a amené à remettre en question sur d'autres plans la représentation malheureuse voulant que I'histoire du Québec contemporain soit placée sous le signe du seul conservatisme clérical. Évidemment, comme la Révolution tranquille est présentée comme le point tournant d'une rupture entre le monde cléricalisé et l'avènement de la modernité, nous avons rédigé un article destiné 
à relativiser cette interprétation en partant des données des recensements du Canada et de recherches à partir de fonds d'archives (Rouillard, 1998, p. 23-51).

L'explication première habituellement fournie pour cerner l'origine de la Révolution tranquille est de nature économique, montrant que la poussée d'industrialisation pendant la Deuxième Guerre mondiale et les années 1950 aurait fait basculer le Québec francophone dans le monde moderne. Mais les données des recensements montrent que les indices $d^{\prime}$ 'industrialisation et d'urbanisation sont comparables entre le Québec et I'Ontario depuis le début du XX siècle. II n'y a pas de retard non plus pour les francophones à s'urbaniser : ils le sont dans les mêmes proportions au Québec que la moyenne canadienne dans les villes de 10000 habitants et plus, de 1921 à 1961 (Rouillard, 1998, p. 31).

En outre, la société francophone comporte une structure sociale diversifiée et elle est traversée par un vigoureux courant de pensée libérale qui anime une portion très importante de la bourgeoisie canadiennefrançaise. Ainsi, au tournant du siècle, la bourgeoisie d'affaires francophone de Montréal accueille à bras ouverts le développement industriel et sécrète une vision matérialiste et individualiste de l'organisation sociale, comme l'a montré Fernande Roy (1988, p. 269275). C'est probablement la même optique qui guide les autres chambres de commerce qui foisonnent au début du siècle et qui n'ont pas été étudiées. Elles forment la Fédération des chambres de commerce de la province de Québec en 1909. Largement réparties dans toute la province, on compte 49 chambres en 1938, soucieuses d'assurer le développement économique de leur ville ou région.

Ces milieux d'affaires sont proches du Parti libéral, qui domine presque complètement la vie politique québécoise au fédéral et au provincial de 1897 à 1936. Plusieurs travaux montrent que l'administration libérale oriente les politiques gouvernementales vers le développement industriel et cherche du mieux qu'elle peut à protéger l'autonomie de l'État à l'égard de l'Église. D'ailleurs, I'administration d'Alexandre Taschereau entretient des relations très tendues avec les autorités religieuses (Dupont, 1972). Cette vision libérale de la société comprend aussi une grande admiration pour le système démocratique britannique, sujet sur lequel nous revenons plus bas. Elle est défendue par les grands quotidiens francophones très proches du Parti libéral qui détiennent des tirages considérables (La Presse, 
La Patrie, Le Soleil). Le journal La Presse, quotidien résolument moderne à tous les points de vue, détient un tirage quotidien atteignant près de 150000 exemplaires en 1920 (Canadian Almanach and Directory, 1921, p. 364-365). À l'encontre de la presse cléricale qui porte un regard hostile à l'endroit de l'industrialisation, ces journaux font la promotion du développement industriel, du système capitaliste et de la participation des francophones à la croissance économique. C'est pour combattre leur orientation que le clergé catholique fonde le quotidien L'Action catholique à Québec en 1907, indirectement Le Devoir en 1910 et Le Droit à Ottawa en 1913.

Quant aux travailleurs québécois, il n'y a pas de retard à prendre conscience de leur condition de salariés et de la nécessité de se regrouper pour défendre leurs intérêts. Comme nous l'avons montré plus haut, la propension à la grève et la densité syndicale sont comparables à celles des autres travailleurs nord-américains depuis le début du XXe siècle. Comme ailleurs en Amérique du Nord, les syndicats internationaux dominent le paysage syndical, imposent une stratégie de relations de travail pour relever la condition ouvrière et implantent dans la province une structure d'intervention auprès des pouvoirs publics.

Enfin, il ne faut pas conclure de notre démarche que la Révolution tranquille est un événement mineur dans I'histoire du Québec et que, pour les décennies antérieures, la province a évolué «normalement» comme les autres sociétés industrielles. Par certains de ses traits, son histoire est distincte dans l'ensemble nord-américain, notamment par la culture française qui I'habite, par la forte influence du clergé catholique et par son statut minoritaire dans l'ensemble canadien. La Révolution tranquille marque une accélération de son histoire qui témoigne de changements sociaux profonds, notamment par la sécularisation de la société et le développement du sens d'identification au Québec. Les années 1960 marquent donc le triomphe du modèle libéral de société ou plutôt social-démocrate, car l'État étend considérablement son champ d'intervention.

\section{LA PENSÉE LIBÉRALE}

Sur le thème du monolithisme idéologique au Québec découlant de l'emprise cléricale, nous avons rédigé deux articles et un autre est 
en chantier pour illustrer que la pensée libérale est loin d'être un courant marginal et qu'elle anime les hommes politiques québécois pendant la période dite de Grande Noirceur. Ils concernent la devise du Québec, le Congrès d'orientation du Parti libéral en 1938 et la construction du pont de Québec.

La devise du Québec, "Je me souviens», veut transmettre un souvenir dont la signification est loin d'être évidente pour la plupart des Québécois et Québécoises. Cette ignorance est le résultat, à mon avis, de la construction historique des artisans de la Révolution tranquille qui ne voit pas de différence entre les orientations politiques du gouvernement de I'Union nationale et celles des gouvernements antérieurs. La formulation de la devise revient à l'architecte du Parlement de Québec, Eugène-Étienne Taché, membre d'une grande famille bourgeoise de la ville de Québec. Lors de l'édification du Parlement, il I'a fait graver vers 1885 dans la pierre au fronton de la porte principale sous les armoiries du Québec. II n'a pas l'intention d'en faire la devise du Québec, mais graduellement les gens l'ont interprétée dans ce sens et elle l'est devenue officiellement en 1939.

La signification de la devise est l'objet de controverses. Taché n'a jamais indiqué clairement le sens de ce qui est devenu la devise du Québec et plusieurs commentateurs ont conclu que la devise devrait se lire ainsi: "Je me souviens que né sous le lys, je crois sous la rose». $C^{\prime}$ est le sens que lui a donné le premier ministre Jean Charest en réponse à une «colle » qu'un étudiant de l'Université des langues étrangères de Pékin lui a posée en 2005 (La Presse: 25 septembre 2005, p. A6). Mais Gaston Deschênes, directeur des études documentaires à l'Assemblée nationale du Québec de 2000 à 2004, rejette cette interprétation. Selon lui, la devise n'a pas de signification particulière: elle peut être interprétée librement et représente une invitation générale au souvenir. Ce serait un mythe que de penser qu'elle puisse évoquer un quelconque bienfait de l'administration britannique (Deschênes, 2002, 2012) ${ }^{7}$.

Nous avons commencé à nous intéresser à la signification de la devise après qu'une secrétaire du département nous ait posé la question à ce sujet. Un historien du Québec ne devrait-il pas connaître le sens de la devise de sa province qui apparaît d'ailleurs sur les plaques d'immatriculation depuis 1978? Nos premières recherches et la visite guidée du Parlement nous ont tout de suite permis de faire un lien avec l'interprétation que 
les historiens en vogue à la fin du XIX siècle se faisaient de l'histoire du Québec: Louis-Philippe Turcotte, Benjamin Sulte, Laurent-Olivier David, Alfred DeCelles et surtout Thomas Chapais. En effet, ils tiennent en haute estime les institutions britanniques parce qu'elles ont permis l'acquisition du système démocratique de gouvernement et une autonomie politique pour le Québec. Grâce à ces institutions, le Canada français, croient-ils, a pu survivre et se développer dans un rapport d'égalité avec les Canadiens anglais. Cette vision se retrouve bien ramassée dans la formule-choc d'Hector Fabre, reprise par le premier ministre Sir Wilfrid Laurier, voulant que la France ait donné au Canada français «le premier des biens, l'existence », mais que "c'est à l'Angleterre que nous devons le second, la liberté »(Lamonde, 2000, p. 461, 486).

Nous avons rédigé deux articles sur la signification de la devise qui fait appel au souvenir et doit être interprétée comme un texte introduisant à I'Hôtel du Parlement lui-même (Rouillard, 2005, p. 127-145; 2007, p. 233-237). Taché, qui est un héraldiste chevronné, conçoit l'édifice comme un «témoin » de l'histoire du Québec, un lieu de mémoire destiné à illustrer de manière symbolique I'identité du Québec. L'ornementation générale de l'Hôtel du Parlement, intérieure et extérieure, devient la clef pour saisir la signification de la devise. Taché recourt fréquemment à la fleur de lys parmi les éléments iconographiques pour rappeler les origines françaises du Québec. Mais plus nombreuses encore sont les références aux symboles royaux britanniques (couronnes, léopards, lions, roses). De plus, les armoiries de la Grande-Bretagne sont placées à des endroits stratégiques et bien en évidence: au faîte de la tour centrale de la façade du Parlement et au-dessus des trônes des Orateurs de l'Assemblée législative et du Conseil législatif. Plus révélateur encore, Taché réserve deux niches pour des statues grandeur nature à gauche et à droite du fauteuil de l'Orateur de l'Assemblée législative à la Reine Victoria et à son mari le Prince Albert. Les deux statues sont encore visibles à la fin des années 1920 et par la suite remplacées par des pots de fleurs.

Enfin, la vision du Québec de Taché s'exprime aussi dans les 22 statues, grandeur nature, posées dans des niches sur la façade du Parlement. Le choix de ces "gloires nationales» n'est pas arbitraire; il correspond aux orientations et aux valeurs que Taché veut mettre en relief. Comme l'édification de ces statues s'est faite graduellement 
jusqu'à la fin des années 1920, les gouvernements qui se sont succédé ont apporté des changements dans le choix des personnages qui ne dérogent pas cependant aux intentions de Taché. Bon nombre d'entre elles illustrent des figures bien connues de la Nouvelle-France: découvreurs, gouverneurs, militaires et missionnaires. C'est la période héroïque visant à célébrer les origines françaises du Québec. Le marquis de Montcalm est du nombre tout comme le vainqueur des Plaines d'Abraham, le général Wolfe, pour les raisons qu'on comprendra plus bas. Pour le régime britannique, on a retenu cinq personnages dont quatre sont liés à la conquête des institutions parlementaires: les gouverneurs lord Dorchester, sous lequel est accordée la Chambre d'Assemblée en 1791, et lord Elgin, qui reconnaît le gouvernement responsable en 1848. Pour souligner encore davantage l'importance de cette dernière avancée qui consacre l'autonomie politique du Parlement sur les affaires internes de la colonie, le gouvernement ajoute en 1920 les statues de Louis-Hippolyte La Fontaine et de Robert Baldwin dont les efforts concertés ont permis cette conquête. C'est le sentiment largement répandu chez les politiciens et les historiens de l'époque qu'ils sont parvenus à obtenir par des moyens parlementaires la liberté politique recherchée par les patriotes de 1837-1838.

Fils d'un des Pères de la Confédération, Taché inscrit donc de manière symbolique dans l'ornementation générale du Parlement les valeurs de la classe politique de l'époque qui tient en haute estime la GrandeBretagne pour avoir accordé le système démocratique de gouvernement et une autonomie politique qui a assuré le développement du Canada français. Pour être plus certain de cette interprétation, nous avons effectué une recherche dans le fonds Eugène-Étienne Taché conservé à $B A n Q$, où nous n'avons pas trouvé d'informations précises à ce sujet (malheureusement son journal personnel manque pour 1883). Mais il est un lien qui nous apparaît très révélateur de la pensée de Taché, car il est responsable de l'illustration de la médaille du tricentenaire de la ville de Québec en 1908. Cette médaille de bonne dimension se trouve conservée dans plusieurs services d'archives à Québec: Musée de la civilisation, Assemblée nationale, ville de Québec ${ }^{8}$. Elle a été portée à notre attention en lisant le livre de l'historien $\mathrm{H}$. V. Nelles de I'Université York de Toronto consacré au tricentenaire de la ville de Québec. II y reproduit une illustration des deux côtés de la médaille (voir la Figure 1) (Nelles, 2003, p. 15) ${ }^{9}$. 

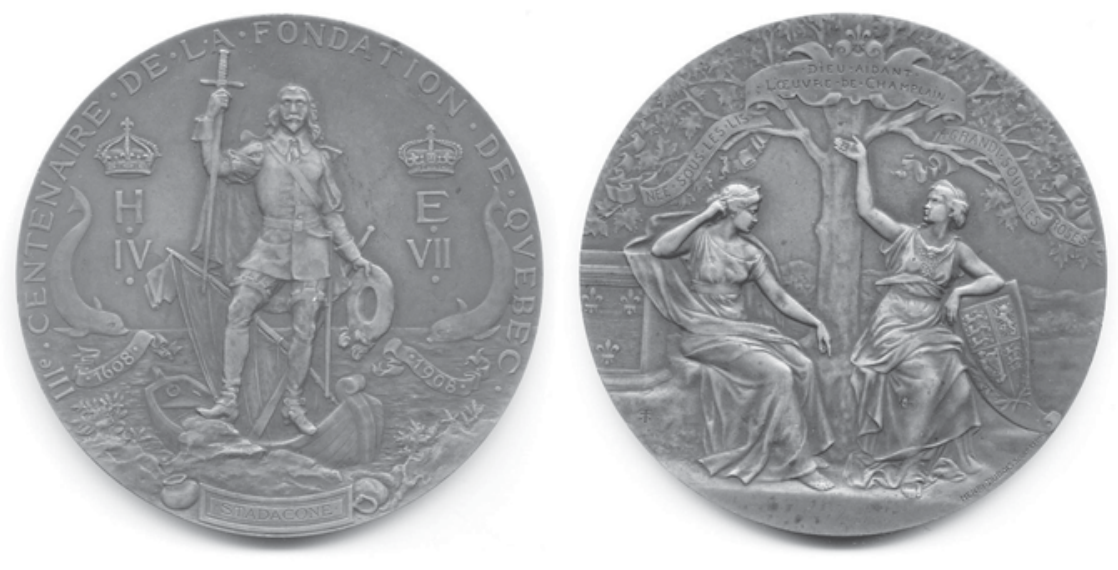

Figure 1. Les deux côtés de la médaille commémorative du III centenaire de la Fondation de Québec en 1907, conçue par Eugène-Étienne Taché et gravée par Henri Dubois.

Source: H. V. Nelles, 2003, p. 15.

Sur une face de la médaille, on retrouve le fondateur de la ville de Québec, Samuel de Champlain, qui débarque à Québec. Mais plus intéressant est le revers où apparaît l'inscription: «Dieu aidant/l'œuvre de Champlain /née sous les lis/a grandi sous les roses ». II s'y trouve deux femmes assises, l'une symbolisant la Nouvelle-France qui pointe le doigt vers le sol, vers les racines d'un arbre qui représente les origines de la ville de Québec. Pour sa part, la muse britannique, appuyée sur l'écu royal de la Grande-Bretagne, porte la main vers le feuillage très fourni de l'arbre composé de feuilles d'érable. Par ces mots et ces symboles, Taché veut illustrer que la présence française s'est épanouie sous l'autorité britannique par tout le Canada. Et il y a fort à parier que les symboles qu'il évoque pour la ville de Québec s'appliquent aussi à la province de Québec. Nelles ne fait pas de lien entre la médaille et la devise, mais le texte et la représentation de la médaille correspondent exactement au souvenir que Taché veut évoquer dans l'inscription qu'il a fait graver au faîte de la porte principale du Parlement et qui est reflétée dans la décoration intérieure et extérieure de l'Hôtel du Parlement.

La préservation de cet artefact par des centres d'archives illustre l'importance du rôle des archivistes dans la préservation de notre mémoire. Finalement, à notre avis, si Taché n'a pas directement précisé 
le sens du «Je me souviens» de son vivant, c'est parce qu'il est décédé en 1912, qu'il ne pensait pas que cette inscription deviendrait la devise du Québec et qu'il lui apparaissait évident qu'il faut lier le texte aux armoiries du Québec et aux thématiques de l'ornementation générale du Parlement qui sont consacrées à la mémoire et à son histoire.

Toujours est-il que la célébration du tricentenaire de Québec, au cours de laquelle la médaille est distribuée, est organisée à l'initiative de la Société Saint-Jean-Baptiste de Québec avec la collaboration du gouverneur général du Canada, lord Albert Grey. Elle donne lieu à deux semaines de manifestations grandioses inaugurées par nul autre que le prince de Galles, futur roi George V, accueilli chaleureusement par la population de Québec. Les adresses de bienvenue à l'égard du prince sont l'occasion de célébrer les bienfaits des institutions britanniques. Selon le maire de Québec, Georges Garneau:

Les Canadiens français éprouvent un sentiment inexprimable d'orgueil patriotique et de reconnaissance envers les deux grandes nations qui ont tour à tour présidé à nos destinées: la France toujours aimée [...]; I'Angleterre, qui les a laissés libres de grandir en gardant leur foi, leur langue et leurs institutions, et qui les a dotés d'un régime constitutionnel fondé sur la plus grande somme de libertés, et qui est, sans contredit, le plus beau et le plus parfait du monde. (Georges Garneau, cité par Roy, 1911, p. 177)

Cette vision de I'histoire du Canada français apparaît étonnante de nos jours, mais elle demeure le point de vue dominant parmi les élites politiques canadiennes-françaises jusqu'à la Deuxième Guerre mondiale. La crise de la conscription pendant la Première Guerre mondiale et la montée d'un courant critique à l'égard de la Confédération et du lien colonial portent un coup dur à célébrer les bienfaits de la monarchie britannique. Cette mémoire est remplacée dans les années 1950 et 1960 par celle des artisans de la Révolution tranquille qui refusent à la bourgeoisie francophone un projet de société autonome et associe ses objectifs à ceux du monde clérical. Tout le passé franco-québécois devient alors teinté d'un conservatisme profond avec comme conséquence la perte de la mémoire de l'appui aux institutions démocratiques par les milieux politiques et par les nombreux journaux liés aux partis conservateur et libéral. Cette vision de I'histoire du Québec porte Pierre Elliott Trudeau 
à conclure que le sens des institutions démocratiques serait étranger aux Canadiens français au point où nous aurions un penchant pour l'autoritarisme et la corruption politique.

\section{L'ORIENTATION SOCIALE-DÉMOCRATE}

Une autre avenue de nos recherches, qui repose en partie sur le fonds Adélard Godbout conservé au Centre d'archives de Québec de BAnQ et sur l'analyse du journal Le Canada, organe du Parti libéral, nous a permis de mettre en évidence la mutation du libéralisme qui anime le Parti libéral du Québec dans les années 1930. La construction de l'histoire du Québec, qui fait tabula rasa du libéralisme des élites politiques, ignore les réalisations de I'administration du Parti libéral d'Adélard Godbout de 1939 à 1944. Elles marquent pourtant un tournant dans l'évolution politique du Québec et représentent une première révolution tranquille qui annonce clairement les réalisations du gouvernement de Jean Lesage dans les années 1960.

Pendant ce seul mandat, le gouvernement Godbout réalise un accomplissement politique remarquable. II subit l'influence des revendications du syndicalisme international, de la vision du New Deal qui s'impose aux États-Unis et de la nouvelle stratégie interventionniste du gouvernement fédéral. Ses réalisations affirment les valeurs démocratiques et une orientation nouvelle, soit d'attribuer à l'État un rôle central pour assurer le développement économique et mettre en place un filet de sécurité sociale. Malgré l'opposition traditionnelle du clergé catholique, il accorde le droit de vote aux femmes en 1940, instaure la gratuité de l'éducation dans les écoles primaires et impose I'instruction obligatoire jusqu'à 14 ans en 1943. Au plan économique, il crée une entreprise d'État en 1941, la Raffinerie de sucre de SaintHilaire, et nationalise la Montreal Light, Heat and Power en 1944, donnant ainsi naissance à Hydro-Québec. L'année précédente, il forme un Conseil d'orientation économique pour planifier la reconversion de l'industrie de guerre.

Au plan social, fidèle à son programme, le gouvernement met en place l'État protecteur en participant en 1940 au programme fédéral d'assurance-chômage comme le réclament les organisations syndicales. Plus encore, il forme la Commission sur les problèmes hospitaliers 
qui recommande en 1943 la mise en place d'un régime provincial et universel d'assurance-maladie. Le gouvernement fait diligence en créant une Commission pour l'instituer, ce qui place le Québec à l'avant-garde des provinces dans cette démarche. Contrairement au gouvernement Duplessis, I'administration Godbout se montre favorable à la syndicalisation en affirmant en 1944 le droit des travailleurs de se syndiquer et de négocier leurs conditions de travail, principes à la base de l'actuel Code du travail.

Malgré ses réalisations impressionnantes, le gouvernement Godbout est défait par l'Union nationale le 30 août 1944, bien qu'il remporte un pourcentage plus élevé du suffrage. L'élection porte non pas sur sa feuille de route, mais sur la conscription imposée par le gouvernement fédéral. Godbout avait promis sur son honneur de combattre son parti si jamais la conscription était instituée. Le gouvernement Duplessis, animé d'une toute autre philosophie politique, s'empresse de démanteler la Commission d'assurance-maladie et le Conseil économique; il réintroduit même la possibilité de droits de scolarité par les commissions scolaires. C'en est fait du coup de barre des politiques gouvernementales vers la modernité jusqu'au retour au pouvoir du Parti libéral en 1960.

Ces réalisations sont connues de nombreux historiens, même si certains ne veulent pas en tenir compte. L'originalité de notre recherche récente tient au coup de barre donné par le Parti libéral à son programme lors de son congrès d'orientation à Québec le 11 juin 1938 (Rouillard, 2015, p. 125-158). Ce congrès, qui réunit plus de mille délégués venant de toutes les régions du Québec, est complètement méconnu. C'est là que le Parti libéral redéfinit son orientation en s'alignant sur la voie de l'État-protecteur et adopte une vision keynésienne de l'État au point de vue économique. Les délégués adoptent des résolutions renforçant les valeurs démocratiques en se prononçant pour la première fois en faveur du droit de vote pour les femmes et pour l'abolition du Conseil législatif, équivalent québécois du Sénat fédéral. Ils jettent aussi les bases d'un système de sécurité sociale en adoptant des résolutions pour participer au programme fédéral d'assurance-chômage, établir un régime québécois de retraite étatique et mettre en vigueur le programme d'aide aux mères sans soutien de famille (Parti libéral du Québec, 1938, p. 42-54). Quoiqu'il n'en soit pas fait mention au congrès, trois autres mesures très importantes apparaissent à la plate-forme électorale du parti l'année suivante: 
l'établissement d'un programme d'assurance-maladie, un programme d'assurance-invalidité et une aide aux familles nombreuses. Élu l'année suivante, le Parti libéral met en branle ces réformes et d'autres que nous avons énumérées plus haut pendant la courte période de quatre ans et neuf mois où il dirige le Québec. C'est un accomplissement remarquable dans une société supposément engluée dans le conservatisme et la «Grande Noirceur».

La consultation du fonds Godbout au Centre d'archives de Québec de BAnQ nous a permis de trouver deux causeries révélatrices de I'évolution de sa philosophie politique et de celle du Parti libéral depuis le début du XX siècle. Elles sont prononcées en 1946 sur les ondes de Radio-Canada alors que Godbout est toujours chef du parti. Pour lui, le libéralisme qui anime le Parti libéral se veut fidèle aux principes fondamentaux développés par des penseurs britanniques, français et américains aux XVII et $\mathrm{XVIII}$ siècles: respect de la liberté individuelle, progrès de l'enseignement public, liberté de religion, liberté d'association et de réunion, liberté de presse, liberté de commerce. "Les libéraux», dit-il, "croient au progrès par la liberté ». Mais son libéralisme, précise-t-il, n'est pas une doctrine «fixée sur le passé»; il est «susceptible d'un éternel rajeunissement». Ainsi, les gouvernements libéraux ont pris leur distance à l'égard du laisser-faire et ont été «les champions de l'autonomie et du progrès social ». Ils sont responsables en Angleterre, au Canada et au Québec des grandes lois sociales pour atténuer les conséquences des crises économiques. Pour Godbout, c'est I'attachement aux valeurs libérales qui a permis à son parti au Québec d'être responsable des progrès accomplis dans le domaine de l'instruction publique (Godbout, 1946).

Ces deux causeries tracent l'évolution idéologique accomplie par le Parti libéral depuis le début du XXe siècle qui a fait reposer fondamentalement sa philosophie sur les idéaux de liberté et de démocratie auxquels se sont rattachées les valeurs sociales-démocrates dans les années 1930. Les opposants au régime de Duplessis dans les années 1950 ignorent complètement cet important courant de pensée même si les réalisations du gouvernement Godbout ne sont pas si éloignées dans le temps, une quinzaine d'années. Le traumatisme duplessiste engendre chez eux une vision réductrice de I'histoire du Québec présentée comme une société monolithique rivée depuis toujours dans le conservatisme clérical. 


\section{LE PONT DE QUÉBEC}

L'interprétation voulant que les élites franco-québécoises valorisent la vie rurale et soient réfractaires à l'industrialisation fait partie de l'imaginaire des partisans de la folk society. C'est ce qui expliquerait leur infériorité économique. Pour appuyer leurs dires, il est souvent cité des extraits d'un sermon prononcé par $\mathrm{M}^{\mathrm{gr}}$ Louis-Adolphe Pâquet de Québec, philosophe ultramontain par excellence, à l'occasion de la célébration de la Saint-Jean-Baptiste en 1902. II porte sur «la vocation de la race française en Amérique » à qui il attribue la mission

moins de manier des capitaux que de remuer des idées [...], moins à allumer le feu des usines qu'à entretenir et à faire rayonner au loin le foyer lumineux de la religion et de la pensée [...]. Laissons à d'autres nations, moins éprises d'idéal, ce mercantilisme fiévreux et ce grossier naturalisme qui les rivent à la matière [...]. (Pâquet, 1902, p. 11 et 21)

Ce sermon est diffusé sous forme de brochure en 1925 et l'extrait cité plus haut est repris ad nauseam comme reflet de la pensée du Canada français à l'égard du commerce et de l'industrie, y compris par Pierre Elliott Trudeau dans le collectif qu'il dirige sur la grève de l'amiante en 1956 (Trudeau, 1956, p. 14).

Mais quand on lit bien le sermon de $M^{\text {gr }}$ Pâquet, ce dernier vise à dénoncer le matérialisme ambiant auquel donne lieu la transformation commerciale et industrielle du Québec: "nous entrons dans une ère de progrès», "l'industrie s'éveille; une vague montante de bien-être, d'activité, de prospérité, envahit nos campagnes» (Pâquet, 1902, p. 20). En effet, la ville de Québec comme tout le Québec d'ailleurs entre dans une phase d'accélération de son industrialisation au début du XX $X^{\mathrm{e}}$ siècle. Comme nous l'avons souligné, une bourgeoisie d'affaires francophones met sur pied des chambres de commerce et les hommes politiques accueillent à bras ouverts le développement industriel.

Le hasard a voulu que nous nous intéressions au pont de Québec à la faveur d'une requête en 2001 pour que nous traitions des conditions de travail des ouvriers employés à sa construction. Nous nous sommes alors demandé pourquoi on avait décidé d'ériger un pont aussi colossal, encore aujourd'hui le plus long pont au monde de structure cantilever, 
« une des merveilles du génie de l'époque». L'ouvrage le plus fouillé sur le pont, celui de Michel L'Hébreux (2001), porte sur l'effondrement du pont en 1907 et 1916 et il ne s'intéresse pas aux facteurs à l'origine de sa construction. Nous avons réalisé une recherche qui est terminée, mais qui n'a pas encore fait l'objet d'une publication, en tirant profit notamment des rapports annuels de fonds de la Chambre de commerce de Québec et autres documents conservés à BAnQ de même que les dossiers de la Division des archives de la Ville de Québec.

Le projet d'édifier un pont est d'abord l'œuvre d'une compagnie privée, la Compagnie du pont de Québec constituée en 1887. La très grande majorité des actionnaires sont francophones et le président fondateur, Simon-Napoléon Parent, est alors maire de Québec (il deviendra premier ministre du Québec de 1900 à 1905). Ce sont les milieux financiers de la ville, notamment les membres de la Chambre de commerce de Québec composés majoritairement de francophones, qui militent pour la réalisation du projet. Inquiets de voir le développement économique de leur ville être dépassé par Montréal, les membres de la Chambre de commerce caressent le programme ambitieux de faire de leur ville la plaque tournante du commerce du blé venant de l'Ouest canadien.

Les Prairies canadiennes se développent alors rapidement et une seule ligne de chemin de fer relie l'Ouest à l'Est canadien, celle du Canadien Pacifique, qui passe à Montréal. Les membres de la Chambre de commerce imaginent la construction d'une seconde ligne plus courte, traversant le nord du Québec et de I'Ontario à partir de Winnipeg. Elle aboutirait à Québec qui verrait l'activité de son port stimulée et devenir un lieu privilégié pour l'exportation du blé en Europe. On prévoit aussi lier le chemin de fer à la ligne Transcontinental qui passe sur la rive sud et se dirige vers la côte Atlantique afin de faciliter l'exportation du blé vers l'Europe. Pour ce faire, il faut l'érection d'un pont enjambant le fleuve.

Sa construction est commencée en 1900 par la Compagnie du pont de Québec. Son financement est appuyé des souscriptions populaires et surtout par les trois paliers de gouvernement. Mais la Compagnie fait faillite en 1907, après l'effondrement du pont alors en construction. Le gouvernement fédéral prend alors en charge son édification qui est terminée en 1917. À propos du chemin de fer, la Chambre de commerce fait pression sur le gouvernement fédéral pour l'édification de la ligne de 
chemin de fer dans le nord du Québec et de l'Ontario. Commencée en 1905, la construction de la ligne, appelée le National Transcontinental, est terminée en 1913. Le rêve des milieux politiques et économiques d'un lien commercial transcontinental se trouve alors concrétisé.

Comment ne pas y voir le dynamisme des milieux d'affaires de la Ville de Québec et la conviction des hommes politiques que le développement de la ville passe par un ambitieux projet de développement économique ${ }^{10}$ ? Le début de la construction du pont de Québec survient au moment même où $\mathrm{M}^{\text {gr }}$ Pâquet récuse l'activité industrielle pour le Canada français et lui attribue la mission de devenir «le foyer lumineux de la religion et de la pensée» (Pâquet, 1902, p. 11). Malheureusement, une vision ratatinée de l'histoire qui se limite à l'idéologie des milieux cléricaux a ignoré le contexte et l'objectif de son discours pour ne retenir que la teneur du message en imaginant que les élites de la société canadienne-française s'y étaient conformées. C'est un autre exemple de l'impact malheureux de la thèse de la folk society.

\section{CONCLUSION}

L'objectif de ce texte est de montrer qu'il existe un fossé entre la mémoire que les Québécois et Québécoises se font de leur passé et sa véritable histoire lorsqu'on tient compte de la diversité de son expérience. La représentation du Québec sous le signe de la «Grande Noirceur avant 1960 » est un mythe qui malheureusement affecte la psychologie collective des Québécois et Québécoises. La mémoire, écrit Joseph Yvon Thériault, est «l'élan du passé qui donne forme au présent et permet la projection dans l'avenir » (Thériault, 2018, p. 159). Rejeter de larges pans de son passé concourt à développer un manque de fierté et d'assurance collectives. C'est d'autant plus dommageable que cette représentation est mal fondée comme plusieurs historiens l'ont démontré. Cependant, elle est tellement enracinée que la recherche historique qui en contredit les assises a du mal à percer dans la mémoire collective à la fois parce que cette vision traditionnelle imprègne les médias, règne sans partage dans les travaux en sciences sociales et s'impose auprès de certains historiens, jeunes et moins jeunes, qui ont un intérêt plus marqué pour I'histoire culturelle axée sur l'étude des idées conservatrices au détriment de I'histoire politique et économique (Kelly, 2003, p. 3-11, 73-91). 
Le tracé humiliant de notre histoire, dressé par des intellectuels dans les années 1950 et 1960, s'inscrit dans leur impatience d'en finir avec le régime Duplessis et son nationalisme réactionnaire quitte à noircir son passé en mettant en évidence son retard et son infériorité. Les sociologues jouent un rôle important dans ce cheminement en empruntant le modèle d'interprétation de la folk society/urban society. La méthodologie de leur discipline, axée sur la conception préalable d'un modèle d'explication, et leur peu d'intérêt pour la recherche historique nous apparaissent à la source de la faiblesse de leur perspective historique.

La recherche historique depuis les années 1970 a revu cette vision de I'histoire du Québec parce que sa méthodologie repose sur une bonne connaissance des faits avant de les interpréter et de construire un récit cohérent. Elle s'est affirmée grâce à un meilleur accès à des instruments de recherche, aux journaux d'époque et aux documents d'archives. L'histoire vraie ne peut se passer du travail des bibliothécaires et des archivistes qui assurent la conservation, le classement et l'accès aux documents du patrimoine écrit. Ils sont en quelque sorte les gardiens de la mémoire d'une société pour éviter que le récit historique devienne une construction mythique qui déforme la réalité et propose un récit sans fondement véritable. La déformation de I'histoire peut avoir des conséquences malheureuses pour une société, car elle affecte l'image qu'elle détient de son identité. Insister pour représenter le passé des Québécois francophones comme une société impuissante, dominée, passive, anachronique, tournée sur elle-même, affecte leur psychologie collective. Malheureusement, c'est encore cette version amputée de notre histoire qui imprègne notre mémoire collective.

JACQUES ROUILLARD 


\section{NOTES DE FIN}

1. Ils ont réuni leurs articles les plus importants sur l'évolution sociale du Québec dans Rioux, M. et Martin, Y. (1971). Pour un bon survol de la sociologie québécoise à ce propos, voir: Warren, J.-P. (2003) et Fournier, M. et Houde, G. (1980).

2. Voir aussi à ce propos: Linteau, P.-A. (2000). Un débat historiographique: I'entrée du Québec dans la modernité et la signification de la Révolution tranquille, dans Bélanger, Y., Comeau, R. et Métivier, C. La Révolution tranquille: 40 ans plus tard, un bilan. Montréal, Québec: VLB éditeur, p. 21-41.

3. Voir notamment la conclusion générale du deuxième volume où les auteurs dégagent leur interprétation de I'histoire du Québec (Linteau, Robert et Ricard, 1986, p. 721-726).

4. Dans un article publié en 2005, Gérard Bouchard commence à réviser son point de vue: il plaide pour une analyse plus nuancée de l'histoire du Québec, remettant même en cause la toute-puissance du clergé catholique. Il en profite cependant pour reprocher aux historiens «modernistes » de «blanchir » notre histoire en "peignant une image d'Épinal de la société francophone mettant en relief sa modernité tous azimuts, son progrès, son urbanisation, son libéralisme, son entrepreneurship, sa diversité idéologique et ethnique ... »(Bouchard, 2005, p. 416).

5. L'idée d'attribuer les origines de la Révolution tranquille à un courant réformiste à l'intérieur de la pensée catholique ou des mouvements d'action catholique dès la fin des années 1930 n'est pas nouvelle. On la retrouve notamment dans un article de Jean-Charles Falardeau en 1965 et dans le volume d'André J. Bélanger en 1977. Elle est reprise dans le volume d'E.-M. Meunier et Jean-Philippe Warren en 2002 et celui de Michael Gauvreau en 2008.

6. Voir pour informations sur le Centre d'histoire et d'archives du travail (CHAT), http://www.archivesquebecoisesdutravail.org/.

7. Nous avons débattu du sens de la devise avec Gaston Deschênes dans le Bulletin d'histoire politique du Québec (Deschênes, 2006; Rouillard, 2007).

8. Nous remercions l'archiviste Gilles Héon d'avoir effectué la recherche pour retrouver les lieux où la médaille est conservée.

9. Nelles a trouvé la médaille aux Archives de l'Ontario dans le fonds de la fille du colonel Denison; elle avait été donnée à son père lors de sa visite à Québec, lors des manifestations du tricentenaire (Archives de I'Ontario, MU2365, Quebec Tercentenery Papers). Nous remercions $\mathrm{H}$. V. Nelles de nous avoir fourni des photos de la médaille du Tricentenaire.

10. Nous avons visité récemment au Musée de la civilisation de Québec la salle d'exposition consacrée au développement économique de la Ville de Québec présentée comme «pôle économique majeur» grâce aux entrepreneurs et aux gens d'affaires. Un ilot 
est consacré au pont de Québec, mais sans qu'il ne soit mentionné qu'il est édifié dans une perspective transcontinentale. Dommage, car il n'y a pas meilleur exemple de vouloir faire de Québec un pôle économique majeur. L'absence de publications sur les motivations qui ont présidé à la construction du pont n'a pas permis de rendre compte des motivations qui ont animé ses promoteurs.

\section{BIBLIOGRAPHIE}

BÉLANGER, A. J. (1977). Ruptures et constantes. Quatre idéologies du Québec en éclatement: La Relève, la JEC, Cité Libre, Parti Pris. Montréal, Québec: Hurtubise/HMH.

BÉLANGER et al. (1973). Les travailleurs québécois, 1851-1896. Montréal, Québec: Les Presses de I'Université du Québec.

BOUCHARD, G. (2000). Genèse des nations et cultures du Nouveau Monde. Montréal, Québec: Boréal.

BOUCHARD, G. (2004). La pensée impuissante. Échecs et mythes nationaux canadiens-français (1850-1960). Montréal, Québec: Boréal.

BOUCHARD, G. (2005). L'imaginaire de la grande noirceur et de la révolution tranquille: fictions identitaires et jeux de mémoire au Québec. Recherches sociographiques, 46(3), 411-436.

CANADIAN ALMANACH AND DIRECTORY. 1921. 364-365. Les tirages des journaux proviennent de l'enquête annuelle de l'Audit Bureau of Circulation.

CARDIN, J.-F. et ROUILLARD, J. (1987). Guide des archives des Unions internationales à Montréal. Montréal, Québec: Université de Montréal.

COUSINEAU, S. (2005, p. A6). Je me souviens... La Presse, A6. Repéré à http://collections.banq.qc.ca/ark:/52327/2202184.

DESCHÊNES, G. (2002). La devise « Je me souviens», site de L'Encyclopédie de l'Agora, 10. Repéré à http://agora.qc.ca/documents/quebec - etat-la_devise_je_me_souviens_par_gaston_deschenes. L'auteur a conservé la même interprétation dans le texte révisé de 2012. 
DESCHÊNES, G. (2006). Le sens original de la devise du Québec: Commentaire sur l'analyse de Jacques Rouillard. Bulletin d'histoire politique, 14(2), 257-261.

DUMONT, F. (1965). La représentation idéologique des classes au Canada français. Recherches sociographiques, 6(1), 13.

DUMONT, F. et al. (dir.) (1974). Idéologies au Canada français, 19001929. Québec: Les Presses de I'Université Laval.

DUPONT, A. (1972). Les relations entre l'Église et l'État sous LouisAlexandre Taschereau, 1920-1936. Montréal, Québec: Guérin.

FALARDEAU, J.-C. (1965). La génération de La Relève, Recherches sociographiques, 6(2), 123-133.

FOURNIER, M. et HOUDE, G. (1980). La sociologie québécoise et son objet: problématique et débats. Sociologie et sociétés, 12(2), 26-31.

GAUVREAU, M. (2008). Les origines catholiques de la Révolution tranquille, 1931-1970. Montréal, Québec: Fides.

GODGOUT, A. (1946). Allocution sur le libéralisme politique au Canada, 25 novembre 1946, 10 p; Causerie sur le libéralisme prononcée à RadioCanada, 30 décembre 1946, 5 p. Fonds Adélard Gordbout (P-712, 4M01-5762, 001297, 001322). BAnQ, Centre d'archives de Québec.

GOSSAGE, P. et LITTLE, J. I. (2015). Histoire du Québec. Entre tradition et modernité. Montréal, Québec: Hurtubise.

GRÉGOIRE, M., MONTIGNY, É. et RIVEST, Y. (2016). Le cœur des Québécois. L'évolution du Québec de 1976 à aujourd'hui. Québec: Presses de l'Université Laval.

HAMEL, R. (1974). Rodolphe Hamel raconte sa vie ouvrière. Vie ouvrière, $82,75$.

HAMELIN, J. et ROBY, Y (1971). Histoire économique du Québec, 18511896. Montréal, Québec: Fides.

HAMELIN, J., LAROCQUE, P. et ROUILLARD, J. (1970). Répertoire des grèves dans la province de Québec au XIX siècle. Montréal, Québec: Les Presses des Hautes Études Commerciales. 
HÉON, G. (1995). Les Archives nationales du Québec: la mémoire de la nation. Archives, 27(2), 1995, 10-12.

KELLY, S. (dir.) (2003). Les idées mènent le Québec. Essais sur une sensibilité historique, Québec. Québec: Les Presses de I'Université Laval.

LAMONDE, Y. (2000). Histoire sociale des idées au Québec, 1760-1896. Montréal, Québec: Fides.

LAMONDE, Y. (2011). La modernité au Québec. La crise de l'homme et de l'esprit, 1929-1939. Montréal, Québec: Fides.

LAMONDE, Y. (2016). La modernité au Québec. La victoire différée du présent sur le passé. Montréal, Québec: Fides.

LAMONDE, Y. (2017). Le coin dans la mémoire. L'hiver de notre mécontentement. Montréal, Québec: Leméac.

LESSARD, R. Compilation des données sur la fréquentation des salles du Pavillon Casault, 1971-2017, Coordonnateur, Section de la Diffusion, Direction des services aux usagers et aux partenaires, BAnQ, Pavillon Louis-Jacques Casault, Québec.

LÉTOURNEAU, J. (2014). Je me souviens? Le passé du Québec dans la conscience de sa jeunesse. Montréal, Québec: Fides.

L'HÉBREUX, M. (2001). Le Pont de Québec. Québec: Éditions du Septentrion.

LINTEAU, P.-A. (2000). Un débat historiographique: I'entrée du Québec dans la modernité et la signification de la Révolution tranquille. Dans Y. Bélanger, R. Comeau et C. Métivier. La Révolution tranquille: 40 ans plus tard, un bilan (p. 21-41). Montréal, Québec: VLB éditeur.

LINTEAU, P.-A., DUROCHER, R. et ROBERT, J.-C. (1979). Histoire du Québec contemporain. De la Confédération à la crise (1867-1929). Montréal, Québec: Boréal Express.

LINTEAU, P.-A., DUROCHER, R., ROBERT, J.-C. et RICARD, F. (1979). Histoire du Québec contemporain. Le Québec depuis 1930. Montréal, Québec: Boréal. 
MEUNIER, E.-M., WARREN, J.-P. (2002). Sortir de la Grande Noirceur. L'horizon personnaliste de la Révolution tranquille. Québec: Septentrion.

NELLES, H. V. (2003). L'Histoire spectacle. Le cas du tricentenaire de Québec. Montréal, Québec: Boréal.

OUELLET, F. (1966) Histoire économique et sociale du Québec, 17601850. Montréal, Québec: Fides.

PÂQUET, M ${ }^{g r}$ L-A. (1902). La vocation de la race française en Amérique. La Bibliothèque électronique du Québec, vol. 113, version 1.0, 11 et 21. Repéré à http://beq.ebooksgratuits.com/pdf/Paquet-vocation.pdf.

PARTI LIBÉRAL DU QUÉBEC. L'historique du congrès libéral des 10 et 11 juin 1938. PLQ, 1938, 42-54.

RIOUX, M. et MARTIN, Y. (1971). La société canadienne-française. Montréal, Québec: Éditions Hurtubise/HMH.

ROUILLARD, J. (1974). L'action politique ouvrière, 1899-1915. Dans DUMONT, F. et al. (dir.), Idéologies au Canada français, 1900-1929 (p. 267-312). Québec: Les Presses de l'Université Laval.

ROUILLARD, J. (1974). Les travailleurs du coton au Québec, 1900-1915. Montréal, Québec: Les Presses de I'Université du Québec.

ROUILLARD, J. (1974). Bilan de la situation des archives syndicales au Québec. Bulletin du Regroupement des chercheurs en histoire des travailleurs québécois, 1(3), 32-38.

ROUILLARD, J. (1974). Les archivistes et I'histoire des travailleurs. Archives 1974, 1974, 67-69.

ROUILLARD, J. (1978). La récupération des archives syndicales au Québec. Archivaria, 7, 125-130.

ROUILLARD, J. (1979). Les syndicats nationaux au Québec, 1900-1930. Québec: Les Presses de I'Université Laval (Prix Lionel-Groulx de I'Institut d'histoire de l'Amérique française, 1980).

ROUILLARD, J. (1983). Le militantisme des travailleurs au Québec et en Ontario: niveau de syndicalisation et mouvement de grèves (1900-1980). Revue d'histoire de l'Amérique française, 37(2), 201-225. 
ROUILLARD, J. (1986). Quebec Report. Canadian Labour Archives Bulletin, $5(3)$.

ROUILLARD, J. (1989). Histoire du syndicalisme québécois. Montréal, Québec: Boréal.

ROUILLARD, J. (1990). Haro sur le fascisme: La fondation de la Fédération provinciale du travail du Québec, 1938. The Canadian Historical Review, 71(3), 346-374.

ROUILLARD, J. (1998). La Révolution tranquille: rupture ou tournant? Revue d'études canadiennes/Journal of Canadian Studies, 32(4), 23-51.

ROUILLARD, J. (1999). La grève de I'amiante. Mythe et symbolique. L'Action nationale, 89(7), 33-43.

ROUILLARD, J. (2000). La grève de l'amiante de 1949 et le projet de réforme de l'entreprise. Comment le patronat a défendu son droit de gérance. Labour/Le Travail, 46, 307-342.

ROUILLARD, J. (2001). De l'importance du syndicalisme international au Québec (1900-1957). Dans Bélanger, Y., Comeau, R. et Métivier, C. (dir.). La FTQ, ses syndicats et la société québécoise (p. 11-36). Montréal, Québec: Comeau \& Nadeau, 2001.

ROUILLARD, J. (2004). Le syndicalisme québécois. Deux siècles d'histoire. Montréal, Québec: Boréal.

ROUILLARD, J. (2005). L'énigme de la devise du Québec: à quel souvenir fait-elle référence? Bulletin d'histoire politique, 13(2), 127-145.

ROUILLARD, J. (2007). Réplique à Gaston Deschênes: La devise du Québec. Bulletin d'histoire politique, 15(2), 233-237.

ROUILLARD, J. (2009). L'expérience syndicale au Québec. Ses rapports avec I'État, la nation et l'opinion publique. Montréal, Québec: VLB éditeur.

ROUILLARD, J. (2010). La fête du Travail à Montréal le premier lundi de septembre, symbole de l'affirmation de la classe ouvrière dans l'espace public (1886-1952). Revue d'histoire de l'Amérique française, 64(2), 33-73. 
ROUILLARD, J. (2011). La fondation du Monde ouvrier/The Labor World, organe du syndicalisme international à Montréal (1916-1941). Bulletin du Regroupement des chercheurs en histoire des travailleurs québécois, 37(1), 19-26.

ROUILLARD, J. (2015). Aux sources de la Révolution tranquille: le congrès d'orientation du Parti libéral du Québec du 10 et 11 juin 1938. Bulletin d'histoire politique, 24(1), 125-158.

ROY, C. (1911). Les fêtes du troisième centenaire de Québec, 1608-1908. Québec: Laflamme.

ROY, F. (1988). Progrès, harmonie, liberté. Le libéralisme des milieux d'affaires francophones à Montréal au tournant du siècle. Montréal, Québec: Boréal.

RUDIN, R. (1992). Revisionism and the Search for a Normal Society: A Critique of Recent Quebec Historical Writing. The Canadian Historical Review, 73(1), 30-61.

RUDIN, R. (1995). La quête d'une société normale. Critique de la réinterprétation de l'histoire du Québec. Bulletin d'histoire politique, $3(2), 9-42$.

THÉRIAULT, J. R (2018). La mémoire comme tradition vivante. Bulletin d'histoire politique, 26(3), 158-164.

TRUDEAU, P. E. (dir.) (1956). La grève de l'amiante. Une étape de la révolution industrielle au Québec. Montréal, Québec: Cité libre.

WARREN, J.-P. (2003). L'engagement sociologique (2003). Montréal, Québec: Boréal. 